
\title{
25 Research Soure \\ Cu-Catalyzed, Mn-Mediated Propargylation and Allenylation of Aldehydes with Propargyl Bromides
}

Rongli Zhang ( $\sim 1543046703 @ q q . c o m$ )

Xuzhou Medical University

Yanping Xia

Gannan Medical University

Yuchen Yan

Xuzhou Medical University

Lu Ouyang

Gannan Medical University

\section{Research Article}

Keywords: Propargylation, Allenylation, Mn powder, Cu-catalyzed, Gram scale

Posted Date: November 24th, 2021

DOI: https://doi.org/10.21203/rs.3.rs-1080113/v1

License: (c) (i) This work is licensed under a Creative Commons Attribution 4.0 International License.

Read Full License

Version of Record: A version of this preprint was published at BMC Chemistry on March 18th, 2022. See the published version at https://doi.org/10.1186/s13065-022-00803-3. 


\section{Abstract}

A simple, practical, and high chemo-selective method for the synthesis of propargyl alcohol and allenyl alcohols via Cu-catalyzed, Mn-mediated propargylation and allenylation of aldehydes with propargyl bromides has been established. When 3-bromo-1-propyne was conducted under the standard condition, the aldehydes were transformed to the corresponding propargylation products completely, while when 1bromo-2-pentyne was used, allenic alcohol was the only product. Variety of homopropargyl alcohols and allenyl alcohols were obtained in high yields and the reaction is compatible with broad substrate scopes. In addition, the large-scale reaction could also be proceeded smoothly indicating the potential synthetic applications of this transformation.

\section{Introduction}

Propargyl and allenyl groups are not only valuable building blocks for further manipulations and organic transformations in organic synthesis, ${ }^{1}$ but also sever as active structural moieties in plentiful functional molecules which are important in bioactive molecules, pharmaceuticals agents and natural products. ${ }^{2}$ Thus, this interesting and promising synthetic method has been attracting a great deal of attentions. ${ }^{3}$ Numerous methods have been established by using propargyl halides and metals to produce the nucleophilic character of the propargyl metal species. ${ }^{4}$ When the nucleophilic receptor is an aldehyde, the homopropargyl alcohol can be obtained by the nucleophilic addition of propargyl metal species and

aldehyde. ${ }^{5}$ Variety of metals, including $\mathrm{In},{ }^{6} \mathrm{Sb},{ }^{7} \mathrm{~Pb},{ }^{8} \mathrm{Ti}^{9} \mathrm{Cr}^{10} \mathrm{Ga}^{11} \mathrm{Sn},{ }^{12} \mathrm{Zn}^{13} \mathrm{Mn},{ }^{14}$ and $\mathrm{Sc}^{15}$, have been used for this coupling reaction which could afford the corresponding homopropargyl alcohols. While, the by-product allenyl alcohol is inevitable, which can be owned to the rearrangement of the crucial intermediate progargyl metal species to allenyl metal species. ${ }^{16}$ Therefore, a mixture of homopropargyl alcohol and allenyl alcohol were generally obtained. Despite the encouraging progress has been made, long reaction time-cost, moderate yields and low chemo-selectivity has limited the applications. Therefore, there is still demands for the improved method with respect to selectivities for homopropargyl alcohol and allenyl alcohols.

As we known, Cu catalyst, is not only abundant, easy to utilize, and relatively insensitive to water and air, but also has advantageous for the controllable access to $\mathrm{Cu}(0), \mathrm{Cu}(\mathrm{I}), \mathrm{Cu}(\mathrm{II})$, and $\mathrm{Cu}(\mathrm{III})$ oxidation states, ${ }^{17}$ possibly because of its single-electron transfer (SET) and two-electron processes (TEPs) pathway, ${ }^{18}$ which make the catalytic system with high catalytic activities and rate. Moreover, Manganese has been widely used in organic reactions by virtue of its environmentally benign and sustainable nature, low cost and versatile reactivity ${ }^{19}$ However, Mn-mediated proparylation reaction was discovered with only few examples. The combination of Cu-catalyst and Mn powder may increase the catalytic efficiency.

In this paper, we developed the first example of Cu-catalyzed and Mn-mediated propargylation and allenylation of aldehydes with propargyl bromides under a novel catalytic system, which is covered with 
advantages of high efficiency, good chemo-selectivity, and wide substrates scopes under mild reaction conditions (Figure 1).

We initiated our investigation using benzaldehyde (1a) and propargyl bromide (2a) as model substrates which catalyzed by copper salts and Mn powder (Table 1). Without Mn, only trace amount of desired product was observed which indicated that Mn powder is indispensable (Table 1, entry 1 ). While in the absence of $\mathrm{CuBr}_{2}, 16 \%$ of $3 \mathbf{a}$ was produced which demonstrated the great importance of $\mathrm{Cu}$ catalyst (entry 2). Screening of different solvents illustrated that $\mathrm{MeCN}$ is the best reaction medium, giving the desired product $3 a$ in $47 \%$ yield (entries 3 ). While, only trace amount of product was observed in THF or DCM and $24 \%$ in EtOH (entries 4-6). The yield of products dropped sharply when the reaction was carried out in the open system (entry 7). Meanwhile, without the addition of $\mathrm{CF}_{3} \mathrm{COOH}$, only $13 \%$ yield of $3 \mathrm{a}$ was achieved (entry 8). Subsequently, extensive experiments were conducted to investigate the effects of different copper salts on the reaction. Series of $\mathrm{Cu}$ catalysts, including $\mathrm{CuSO}_{4}, \mathrm{CuCl}, \mathrm{CuCl}_{2}, \mathrm{CuBr}$ and $\mathrm{Cul}$ were tested and $\mathrm{CuCl}$ gave the best result (entries 9-13). Adding 5 equiv. Mn powder, a remarkable increase has been presented (entry 14). Simultaneously, a light increase of yield was observed by increasing the amount of catalyst (entry 15). Further studies indicated that extending the reaction time to $24 \mathrm{~h}, 1 \mathrm{a}$ can be transformed to $3 \mathrm{a}$ completely under the standard conditions (entry 16).

With the optimized setup in hand, we next explored the substrates scope of aldehydes with different functional groups as shown in Table 2. It is pleasing that substrates bearing both electron-donating groups (EDGs) and electron-withdrawing groups (EWGs) can proceed smoothly. For example, substrates $\mathbf{3 c}, \mathbf{3 e}, \mathbf{3} \mathbf{f}, \mathbf{3 g}, \mathbf{3 h}, \mathbf{3} \mathbf{i}$ and $\mathbf{3 k}$ with alkyl and alkoxy groups can be transformed to the corresponding products in excellent yield. Substrates containing the halogen (3b, $\mathbf{3} \mathbf{d}, \mathbf{3 i}, \mathbf{3} \mathbf{j})$ can also deliver the corresponding products with excellent yields. In addition, disubstituted benzaldehydes, such as 2,4dimethyl (3l), 2,3-dimethyl (3o), 2,5-difluoro (3m), 2,3-difluoro (3n), 2-methoxy-4-methyl (3p) 3-chloro-5fluoro (3q), 3-methoxy-4-fluoro (3r) and 3-methyl-4-fluor (3s) benzaldehydes were found to be compatible with the reaction in $85 \%-95 \%$ yields. To further expand the scopes of the present catalytic system, reactions of heteroaromatic aldehydes including thiophenecarboxaldehyde (3u), pyridylaldehydes ( $3 \mathbf{v}$ and $\mathbf{3 w}$ ) and quinolinecarboxaldehyde (3x) which contain aromatic heterocycle in the molecules were also explored. Interesting, all of these substrates were compatible with the reaction conditions and produced the homopropargyl alcohols in excellent yield. Naphthyl compounds is also effective for the transformation converted to $3 \mathbf{y}$ and $\mathbf{3 z}$ in the yield of $94 \%$ and $96 \%$ respectively.

When 1-bromo-2-pentyne (4a) was used instead of propargyl bromide, the rearrangement product allenyl alcohol was achieved with good yield under the same reaction conditions (Table 3). Importantly, the direct propargylation product was not detected in this catalytic system, which indicated that the chemoselectivity for this reaction is quite good. For example, substrates which substituted by isopropyl-(5a), methyl-(5b) and fluoro-(5c) groups on the aromatic ring, reacted well and provided the corresponding products in moderate yields. In addition, heteroaromatic aldehyde is also worked for the transformation and an allenyl substituted alcohol (5e) was obtained with $85 \%$ yield. 
To demonstrate the synthetic applications of our protocols, we tried to scale up the reaction of benzaldehyde (1a) with 3-bromo-1-propyne (2a) or 1-bromo-2-pentyne (4a) independently under standard conditions (Figure 2). The corresponding products $\mathbf{3} \mathbf{a}$ or $\mathbf{5} \mathbf{a}$ was obtained in a gram-scale, which highlightened the potential applicability of this transformation in organic synthesis.

\section{Experimental}

\section{Procedure for the synthesis of homopropargyl alcohol}

In a $10 \mathrm{~mL}$ Schlenk tube, a mixture of aldehyde $(0.5 \mathrm{mmol})$, was added to a stirred solution of 3-bromo-1propyne (1.5 eq.), $\mathrm{CuCl}$ (10 mol\%), $\mathrm{Mn}$ powder (3.0 eq.), $\mathrm{CF}_{3} \mathrm{COOH}(25 \mathrm{~mol} \%)$ and $\mathrm{MeCN}(2 \mathrm{~mL})$ at room temperature under $\mathrm{N}_{2}$ atmosphere. After 24 hours, the mixture was extracted with EtOAc $(3 \times 10 \mathrm{~mL})$. The combined EtOAc layer was distilled and the crude product was then purified via column chromatograph.

\section{Procedure for the synthesis of allenyl alchols}

In a $10 \mathrm{~mL}$ Schlenk tube, a mixture of aldehyde $(0.5 \mathrm{mmol})$, was added to a stirred solution of 1-bromo-2pentyne (1.5 eq.), $\mathrm{CuCl}$ (10 mol\%), $\mathrm{Mn}$ powder (3.0 eq.), $\mathrm{CF}_{3} \mathrm{COOH}(25 \mathrm{~mol} \%)$ and $\mathrm{MeCN}(2 \mathrm{~mL})$ at room temperature under $\mathrm{N}_{2}$ atmosphere. After 24 hours, the mixture was extracted with EtOAc $(3 \times 10 \mathrm{~mL})$. The combined EtOAc layer was distilled and the crude product was then purified via column chromatograph.

1-phenylbut-3-yn-1-ol (3a) ${ }^{20} \mathrm{~d} 98 \%$ yield $(71.6 \mathrm{mg})$, colourless oil. ${ }^{1} \mathrm{H} \mathrm{NMR}\left(400 \mathrm{MHz}, \mathrm{CDCl}_{3}\right) \delta$ 7.46-7.34 ( $\mathrm{m}$, $4 \mathrm{H}), 7.30(\mathrm{ddd}, \mathrm{J}=8.5,3.6,1.6 \mathrm{~Hz}, 1 \mathrm{H}), 4.88(\mathrm{t}, \mathrm{J}=5.4 \mathrm{~Hz}, 1 \mathrm{H}), 2.71-2.56(\mathrm{~m}, 2 \mathrm{H}), 2.45(\mathrm{~s}, 1 \mathrm{H}), 2.19-1.96$ $(\mathrm{m}, 1 \mathrm{H}) ;{ }^{13} \mathrm{C}$ NMR $\left(100 \mathrm{MHz}, \mathrm{CDCl}_{3}\right) \delta 142.4,128.5,128.0,125.8,80.7,72.3,71.0,29.5$.

1-(4-chlorophenyl)but-3-yn-1-ol (3b) ${ }^{20} \square 96 \%$ yield $(86.7 \mathrm{mg})$, colorless oil. ${ }^{1} \mathrm{H}$ NMR $(400 \mathrm{MHz}$, $\left.\mathrm{CDCl}_{3}\right)$ ठ 7.33-7.25 (m, 4H), $4.80(\mathrm{t}, \mathrm{J}=5.1 \mathrm{~Hz}, 1 \mathrm{H}), 2.81(\mathrm{~s}, 1 \mathrm{H}), 2.58(\mathrm{dd}, \mathrm{J}=6.4,2.5 \mathrm{~Hz}, 2 \mathrm{H}), 2.06(\mathrm{dd}, \mathrm{J}=$ $3.4,1.7 \mathrm{~Hz}, 1 \mathrm{H}) .{ }^{13} \mathrm{C} \mathrm{NMR}\left(100 \mathrm{MHz}, \mathrm{CDCl}_{3}\right) \delta 140.9,133.7,128.6,127.2,80.3,71.6,71.4,29.4$.

1-(p-tolyl)but-3-yn-1-ol (3c) ${ }^{20} \square 91 \%$ yield $(72.8 \mathrm{mg})$, colorless oil. ${ }^{1} \mathrm{H}$ NMR $\left(400 \mathrm{MHz}, \mathrm{CDCl}_{3}\right) \delta 7.25$ (d, J = $7.7 \mathrm{~Hz}, 2 \mathrm{H}), 7.14$ (d, J = 7.7 Hz, 2H), $4.79(\mathrm{~s}, 1 \mathrm{H}), 2.58$ (dd, J = 11.1, 8.7 Hz, 3H), $2.33(\mathrm{~s}, 3 \mathrm{H}), 2.03(\mathrm{~s}, 1 \mathrm{H})$. ${ }^{13} \mathrm{C}$ NMR $\left(100 \mathrm{MHz}, \mathrm{CDCl}_{3}\right) \delta 139.6,137.7,129.2,125.8,80.9,72.2,70.9,29.3,21.2$.

1-(4-fluorophenyl)but-3-yn-1-ol (3d) ${ }^{20} \mathrm{q} 97 \%$ yield $(79.6 \mathrm{mg})$, colorless oil. ${ }^{1} \mathrm{H}$ NMR $(400 \mathrm{MHz}$, $\left.\mathrm{CDCl}_{3}\right)$ ठ 7.46-7.32 (m, 2H), $7.05(\mathrm{t}, \mathrm{J}=8.7 \mathrm{~Hz}, 2 \mathrm{H}), 4.86(\mathrm{t}, \mathrm{J}=5.5 \mathrm{~Hz}, 1 \mathrm{H}), 2.62(\mathrm{dd}, \mathrm{J}=6.3,2.6 \mathrm{~Hz}, 2 \mathrm{H})$, $2.49(\mathrm{~d}, \mathrm{~J}=2.5 \mathrm{~Hz}, 1 \mathrm{H}), 2.08(\mathrm{t}, \mathrm{J}=2.6 \mathrm{~Hz}, 1 \mathrm{H}) .{ }^{13} \mathrm{C} \mathrm{NMR}\left(100 \mathrm{MHz}, \mathrm{CDCl}_{3}\right) \delta 162.4(\mathrm{~d}, \mathrm{~J}=245 \mathrm{~Hz}), 138.2$ $(\mathrm{d}, \mathrm{J}=3 \mathrm{~Hz}), 127.5 \mathrm{~d}, \mathrm{~J}=8 \mathrm{~Hz}), 115.4(\mathrm{~d}, \mathrm{~J}=21 \mathrm{~Hz}), 80.4,71.7,71.2,29.6$.

1-(4-methoxyphenyl)but-3-yn-1-ol (3e) ${ }^{20} \square 89 \%$ yield (78.4 mg), colorless oil. ${ }^{1} \mathrm{H}$ NMR (400 MHz, $\left.\mathrm{CDCl}_{3}\right) \delta 7.29(\mathrm{~d}, \mathrm{~J}=8.6 \mathrm{~Hz}, 2 \mathrm{H}), 6.95-6.80(\mathrm{~m}, 2 \mathrm{H}), 4.80(\mathrm{t}, \mathrm{J}=6.4 \mathrm{~Hz}, 1 \mathrm{H}), 3.79(\mathrm{~s}, 3 \mathrm{H}), 2.64-2.58(\mathrm{~m}, 2 \mathrm{H})$, 
$2.05(\mathrm{t}, \mathrm{J}=2.6 \mathrm{~Hz}, 1 \mathrm{H}) .{ }^{13} \mathrm{C} \mathrm{NMR}\left(100 \mathrm{MHz}, \mathrm{CDCl}_{3}\right) \delta 159.3,134.8,127.1,113.9,80.9,72.0,70.9,55.3$, 29.3.

1-(4-isopropylphenyl)but-3-yn-1-ol (3f) ${ }^{20} \mathrm{\square} 89 \%$ yield (83.7 mg), colorless oil. ${ }^{1} \mathrm{H} \mathrm{NMR}(400 \mathrm{MHz}$, $\left.\mathrm{CDCl}_{3}\right) \delta 7.30(\mathrm{~d}, \mathrm{~J}=8.1 \mathrm{~Hz}, 2 \mathrm{H}), 7.21(\mathrm{~d}, \mathrm{~J}=8.2 \mathrm{~Hz}, 2 \mathrm{H}), 4.82(\mathrm{~s}, 1 \mathrm{H}), 2.90(\mathrm{dt}, \mathrm{J}=13.8,6.9 \mathrm{~Hz}, 1 \mathrm{H}), 2.62$ $(\mathrm{dd}, \mathrm{J}=6.4,2.6 \mathrm{~Hz}, 2 \mathrm{H}), 2.51(\mathrm{~s}, 1 \mathrm{H}), 2.06(\mathrm{t}, \mathrm{J}=2.6 \mathrm{~Hz}, 1 \mathrm{H}), 1.24(\mathrm{~d}, \mathrm{~J}=6.9 \mathrm{~Hz}, 6 \mathrm{H}) .{ }^{13} \mathrm{C}$ NMR $(100 \mathrm{MHz}$, $\mathrm{CDCl}_{3}$ ) $\delta 148.7,139.9,126.6,125.8,81.0,72.3,70.9,33.9,29.3,24.0$.

1-(3-methoxyphenyl)but-3-yn-1-ol (3g) ${ }^{20} \mathrm{~d} 95 \%$ yield (83.6 mg), colorless oil. ${ }^{1} \mathrm{H}$ NMR (400 MHz, $\left.\mathrm{CDCl}_{3}\right) \delta 7.27(\mathrm{dd}, \mathrm{J}=10.3,5.9 \mathrm{~Hz}, 1 \mathrm{H}), 6.99-6.93(\mathrm{~m}, 2 \mathrm{H}), 6.84(\mathrm{ddd}, \mathrm{J}=8.2,2.5,1.0 \mathrm{~Hz}, 1 \mathrm{H}), 4.85(\mathrm{t}, \mathrm{J}=$ $6.3 \mathrm{~Hz}, 1 \mathrm{H}), 3.81(\mathrm{~s}, 3 \mathrm{H}), 2.69-2.59(\mathrm{~m}, 2 \mathrm{H}), 2.51(\mathrm{~s}, 1 \mathrm{H}), 2.08(\mathrm{t}, \mathrm{J}=2.6 \mathrm{~Hz}, 1 \mathrm{H}) .{ }^{13} \mathrm{C} \mathrm{NMR}(100 \mathrm{MHz}$, $\left.\mathrm{CDCl}_{3}\right) \delta 159.7,144.2,129.6,118.1,113.5,111.3,80.7,72.3,71.0,55.3,29.4$.

1-(m-tolyl)but-3-yn-1-ol (3h) ${ }^{20} \square 83 \%$ yield $(66.5 \mathrm{mg})$, colorless oil. ${ }^{1} \mathrm{H} \mathrm{NMR}\left(400 \mathrm{MHz}, \mathrm{CDCl}_{3}\right) \delta 7.24(\mathrm{t}, \mathrm{J}=$ $7.5 \mathrm{~Hz}, 1 \mathrm{H}), 7.21-7.14(\mathrm{~m}, 2 \mathrm{H}), 7.10(\mathrm{~d}, \mathrm{~J}=7.4 \mathrm{~Hz}, 1 \mathrm{H}), 4.82(\mathrm{t}, \mathrm{J}=6.4 \mathrm{~Hz}, 1 \mathrm{H}), 2.62$ (dd, J = 6.4, $2.6 \mathrm{~Hz}$, $2 \mathrm{H}), 2.51(\mathrm{~s}, 1 \mathrm{H}), 2.35(\mathrm{~s}, 3 \mathrm{H}), 2.06(\mathrm{t}, \mathrm{J}=2.6 \mathrm{~Hz}, 1 \mathrm{H}) .{ }^{13} \mathrm{C} \mathrm{NMR}\left(100 \mathrm{MHz}, \mathrm{CDCl}_{3}\right) \delta 142.5,138.2,128.8$, $128.4,126.4,122.9,80.9,72.4,70.9,29.4,21.5$.

1-(2-chlorophenyl)but-3-yn-1-ol (3i) ${ }^{20} \mathrm{D} 96 \%$ yield $(86.4 \mathrm{mg})$, colorless oil. ${ }^{1} \mathrm{H}$ NMR $\left(400 \mathrm{MHz}, \mathrm{CDCl}_{3}\right) \delta 7.62$ (dd, J = 7.7, 1.4 Hz, 1H), 7.36-7.26 (m, 2H), 7.26-7.20 (m, 1H), $5.28(\mathrm{dd}, \mathrm{J}=7.8,4.0 \mathrm{~Hz}, 1 \mathrm{H}), 2.80$ (ddd, J = 16.9, 3.9, $2.7 \mathrm{~Hz}, 1 \mathrm{H}), 2.69(\mathrm{~s}, 1 \mathrm{H}), 2.54(\mathrm{ddd}, \mathrm{J}=16.9,7.8,2.6 \mathrm{~Hz}, 1 \mathrm{H}), 2.10(\mathrm{t}, \mathrm{J}=2.6 \mathrm{~Hz}, 1 \mathrm{H}) .{ }^{13} \mathrm{C} \mathrm{NMR}$ $\left(100 \mathrm{MHz}, \mathrm{CDCl}_{3}\right) \delta 139.7,131.7,129.4,129.0,127.1,127.1,80.3,71.2,68.7,27.7$.

1-(2-fluorophenyl)but-3-yn-1-ol (3j) ${ }^{20} \mathrm{D} 95 \%$ yield $(79.9 \mathrm{mg})$, colorless oil. ${ }^{1} \mathrm{H} \mathrm{NMR}\left(400 \mathrm{MHz}, \mathrm{CDCl}_{3}\right) \delta 7.52$ (td, J = 7.5, 1.5 Hz, 1H), 7.26 (ddd, J = 7.1, 4.6, 1.9 Hz, 1H), 7.16 (td, J = 7.5, 0.8 Hz, 1H), 7.02 (ddd, J = 10.4, 8.2, $0.9 \mathrm{~Hz}, 1 \mathrm{H}$ ), 5.18 (dd, J = 7.2, $4.9 \mathrm{~Hz}, 1 \mathrm{H}$ ), 2.74 (ddd, J = 16.8, 4.7, $2.6 \mathrm{~Hz}, 1 \mathrm{H}$ ), 2.62 (ddd, J = 16.8, 7.6, $2.6 \mathrm{~Hz}, 2 \mathrm{H}), 2.07(\mathrm{t}, \mathrm{J}=2.6 \mathrm{~Hz}, 1 \mathrm{H}) .{ }^{13} \mathrm{C} \mathrm{NMR}\left(100 \mathrm{MHz}, \mathrm{CDCl}_{3}\right) \delta 160.0(\mathrm{~d}, \mathrm{~J}=244 \mathrm{~Hz}), 129.5$, $129.3(\mathrm{~d}, \mathrm{~J}=8 \mathrm{~Hz}), 127.2$ (d, J = $4 \mathrm{~Hz}), 124.3$ (d, J = $3 \mathrm{~Hz}), 115.3$ (d, J = $22 \mathrm{~Hz}), 80.3,71.1,66.4$ (d, J = 2 $\mathrm{Hz}), 28.2$.

1-(4-propoxyphenyl)but-3-yn-1-ol (3k) ${ }^{20} \square 85 \%$ yield (86.8 mg), colorless oil. ${ }^{1} \mathrm{H}$ NMR (400 MHz, $\left.\mathrm{CDCl}_{3}\right) \delta 7.30(\mathrm{~d}, \mathrm{~J}=8.5 \mathrm{~Hz}, 2 \mathrm{H}), 6.88(\mathrm{~d}, \mathrm{~J}=8.5 \mathrm{~Hz}, 2 \mathrm{H}), 4.83(\mathrm{t}, \mathrm{J}=6.2 \mathrm{~Hz}, 1 \mathrm{H}), 3.91(\mathrm{t}, \mathrm{J}=6.6 \mathrm{~Hz}, 2 \mathrm{H})$, 2.68-2.58 (m, 2H), $2.36(\mathrm{~s}, 1 \mathrm{H}), 2.07(\mathrm{~d}, \mathrm{~J}=2.3 \mathrm{~Hz}, 1 \mathrm{H}), 1.80$ (dd, J = 14.1, 7.0 Hz, 2H), 1.03 (t, J = 7.4 Hz, $3 \mathrm{H}) .{ }^{13} \mathrm{C} \mathrm{NMR}\left(100 \mathrm{MHz}, \mathrm{CDCl}_{3}\right) \delta 158.9,134.4,127.0,114.4,80.9,72.1,70.8,69.5,29.4,22.6,10.5$.

1-(2,4-dimethylphenyl)but-3-yn-1-ol (3l) ${ }^{20} \mathrm{\square} 85 \%$ yield (74.0 mg), colorless oil. ${ }^{1} \mathrm{H}$ NMR (400 MHz, $\left.\mathrm{CDCl}_{3}\right) \delta 7.36(\mathrm{~d}, \mathrm{~J}=7.9 \mathrm{~Hz}, 1 \mathrm{H}), 7.03(\mathrm{~d}, \mathrm{~J}=7.7 \mathrm{~Hz}, 1 \mathrm{H}), 6.95(\mathrm{~s}, 1 \mathrm{H}), 5.04(\mathrm{t}, \mathrm{J}=6.4 \mathrm{~Hz}, 1 \mathrm{H}), 2.62-2.54(\mathrm{~m}$, 2H), $2.45(\mathrm{~d}, \mathrm{~J}=4.8 \mathrm{~Hz}, 1 \mathrm{H}), 2.30(\mathrm{~s}, 3 \mathrm{H}), 2.29(\mathrm{~s}, 3 \mathrm{H}), 2.05(\mathrm{t}, \mathrm{J}=2.6 \mathrm{~Hz}, 1 \mathrm{H}) .{ }^{13} \mathrm{C} \mathrm{NMR}\left(100 \mathrm{MHz}, \mathrm{CDCl}_{3}\right)$ $\delta 137.6,137.4,134.6,131.3,127.0,125.1,81.1,70.7,68.8,28.3,21.0,19.0$. 
1-(2,5-difluorophenyl)but-3-yn-1-ol (3m) ${ }^{20} \mathrm{d9} 9 \%$ yield $(85.6 \mathrm{mg})$, colorless oil. ${ }^{1} \mathrm{H}$ NMR $(400 \mathrm{MHz}$, $\left.\mathrm{CDCl}_{3}\right)$ \& $7.26(\mathrm{ddd}, \mathrm{J}=8.8,5.8,3.0 \mathrm{~Hz}, 1 \mathrm{H}), 7.10-6.79(\mathrm{~m}, 2 \mathrm{H}), 5.24-5.07(\mathrm{~m}, 1 \mathrm{H}), 2.83-2.48(\mathrm{~m}, 3 \mathrm{H}), 2.10$ $(\mathrm{t}, \mathrm{J}=2.6 \mathrm{~Hz}, 1 \mathrm{H}) .{ }^{13} \mathrm{C} \mathrm{NMR}\left(100 \mathrm{MHz}, \mathrm{CDCl}_{3}\right) \delta 158.9(\mathrm{dd}, \mathrm{J}=241,2 \mathrm{~Hz}), 155.3(\mathrm{dd}, \mathrm{J}=238,3 \mathrm{~Hz}), 131.2$ (dd, J = 16, $7 \mathrm{~Hz}$ ), 116.3 (dd, J = 24, $8 \mathrm{~Hz}$ ), 115.5 (dd, J = 24, $9 \mathrm{~Hz}), 113.9$ (dd, J = 25, $4 \mathrm{~Hz}), 79.7,71.6$, 65.9, $28.2(\mathrm{~d}, \mathrm{~J}=1 \mathrm{~Hz})$.

1-(2,3-difluorophenyl)but-3-yn-1-ol (3n) ${ }^{20} 087 \%$ yield (79.2 mg), colorless oil. ${ }^{1} \mathrm{H}$ NMR (400 MHz, $\left.\mathrm{CDCl}_{3}\right)$ 反 7.33-7.23 (m, 1H), 7.18-7.01 (m, 2H), $5.19(\mathrm{dd}, \mathrm{J}=6.9,5.2 \mathrm{~Hz}, 1 \mathrm{H}), 2.82(\mathrm{~s}, 1 \mathrm{H}), 2.74(\mathrm{ddd}, \mathrm{J}=$ $16.8,4.8,2.6 \mathrm{~Hz}, 1 \mathrm{H}), 2.63(\mathrm{ddd}, \mathrm{J}=16.8,7.4,2.5 \mathrm{~Hz}, 1 \mathrm{H}), 2.08(\mathrm{t}, \mathrm{J}=2.5 \mathrm{~Hz}, 1 \mathrm{H}) .{ }^{13} \mathrm{C} \mathrm{NMR}(100 \mathrm{MHz}$, $\mathrm{CDCl}_{3}$ ) $\delta 150.2(\mathrm{dd}, \mathrm{J}=246,12 \mathrm{~Hz}), 147.6(\mathrm{dd}, \mathrm{J}=246,13), 131.9(\mathrm{~d}, \mathrm{~J}=10 \mathrm{~Hz}), 124.2(\mathrm{dd}, \mathrm{J}=7,5 \mathrm{~Hz})$, $121.8(\mathrm{t}, \mathrm{J}=3 \mathrm{~Hz}), 116.5(\mathrm{~d}, \mathrm{~J}=2 \mathrm{~Hz}), 79.8,71.4,66.0(\mathrm{t}, \mathrm{J}=2 \mathrm{~Hz}), 28.2$.

1-(2,3-dimethylphenyl)but-3-yn-1-ol (30) ${ }^{20} \mathrm{\square} 87 \%$ yield $(75.7 \mathrm{mg})$, colorless oil. ${ }^{1} \mathrm{H}$ NMR (400 MHz, $\left.\mathrm{CDCl}_{3}\right) \delta 7.36(\mathrm{~d}, \mathrm{~J}=7.4 \mathrm{~Hz}, 1 \mathrm{H}), 7.17-7.04(\mathrm{~m}, 2 \mathrm{H}), 5.15(\mathrm{dd}, \mathrm{J}=7.6,5.0 \mathrm{~Hz}, 1 \mathrm{H}), 2.61-2.51(\mathrm{~m}, 2 \mathrm{H}), 2.28$ (s, 3H), $2.22(\mathrm{~s}, 3 \mathrm{H}), 2.07(\mathrm{~d}, \mathrm{~J}=2.4 \mathrm{~Hz}, 1 \mathrm{H}), 1.97(\mathrm{~s}, 1 \mathrm{H}) .{ }^{13} \mathrm{C} \mathrm{NMR}\left(100 \mathrm{MHz}, \mathrm{CDCl}_{3}\right) \delta 140.4,137.0,133.2$, $129.4,125.8,122.9,81.2,70.7,69.3,28.3,20.7,14.7$.

1-(2-methoxy-4-methylphenyl)but-3-yn-1-ol (3p) ${ }^{20} \square 85 \%$ yield (80.8 mg), colorless oil. ${ }^{1} \mathrm{H}$ NMR (400 MHz, $\left.\mathrm{CDCl}_{3}\right) \delta 7.25(\mathrm{~d}, \mathrm{~J}=7.6 \mathrm{~Hz}, 1 \mathrm{H}), 6.77(\mathrm{~d}, \mathrm{~J}=7.6 \mathrm{~Hz}, 1 \mathrm{H}), 6.68(\mathrm{~s}, 1 \mathrm{H}), 5.09-4.96(\mathrm{~m}, 1 \mathrm{H}), 3.83(\mathrm{~d}, \mathrm{~J}=6.7$ $\mathrm{Hz}, 3 \mathrm{H}), 2.98(\mathrm{~s}, 1 \mathrm{H}), 2.67$ (dddd, $\mathrm{J}=24.2,10.1,6.3,2.6 \mathrm{~Hz}, 2 \mathrm{H}), 2.34(\mathrm{~s}, 3 \mathrm{H}), 2.03(\mathrm{t}, \mathrm{J}=2.6 \mathrm{~Hz}, 1 \mathrm{H}) .{ }^{13} \mathrm{C}$ $\operatorname{NMR}\left(100 \mathrm{MHz}, \mathrm{CDCl}_{3}\right) \delta 156.2,138.9,127.4,126.8,121.2,111.4,81.5,70.4,68.9,55.2,27.5,21.6$.

1-(3-chloro-5-fluorophenyl)but-3-yn-1-ol (3q) ${ }^{20} \mathrm{d9} 5 \%$ yield (94.1 mg), colorless oil. ${ }^{1} \mathrm{H}$ NMR $(400 \mathrm{MHz}$, $\left.\mathrm{CDCl}_{3}\right) \delta 7.19(\mathrm{~s}, 1 \mathrm{H}), 7.07-6.99(\mathrm{~m}, 2 \mathrm{H}), 4.84(\mathrm{t}, \mathrm{J}=4.6 \mathrm{~Hz}, 1 \mathrm{H}), 2.65-2.61(\mathrm{~m}, 1 \mathrm{H}), 2.59(\mathrm{dd}, \mathrm{J}=6.5,3.0 \mathrm{~Hz}$, $1 \mathrm{H}), 2.11(\mathrm{t}, \mathrm{J}=2.6 \mathrm{~Hz}, 1 \mathrm{H}), 1.68(\mathrm{~s}, 1 \mathrm{H}) .{ }^{13} \mathrm{C} \mathrm{NMR}\left(100 \mathrm{MHz}, \mathrm{CDCl}_{3}\right) \delta 163.7(\mathrm{~d}, \mathrm{~J}=248 \mathrm{~Hz}), 146.2(\mathrm{~d}, \mathrm{~J}=$ $7 \mathrm{~Hz}), 135.1(\mathrm{~d}, \mathrm{~J}=10 \mathrm{~Hz}), 121.9(\mathrm{~d}, \mathrm{~J}=4 \mathrm{~Hz}), 115.6(\mathrm{~d}, \mathrm{~J}=25 \mathrm{~Hz}), 111.4(\mathrm{~d}, \mathrm{~J}=22 \mathrm{~Hz}), 79.6,71.8,71.2$ (d, $\mathrm{J}=2 \mathrm{~Hz}), 29.4$.

1-(4-fluoro-3-methoxyphenyl)but-3-yn-1-ol (3r) ${ }^{20} \square 88 \%$ yield (85.4 mg), colorless oil. ${ }^{1} \mathrm{H}$ NMR (400 MHz, $\mathrm{CDCl}_{3}$ ) $\delta$ 7.09-7.01 (m, 2H), $6.88(\mathrm{ddd}, \mathrm{J}=8.3,4.3,2.1 \mathrm{~Hz}, 1 \mathrm{H}), 4.83(\mathrm{t}, \mathrm{J}=6.3 \mathrm{~Hz}, 1 \mathrm{H}), 3.89(\mathrm{~d}, \mathrm{~J}=5.9 \mathrm{~Hz}$, $3 \mathrm{H}), 2.62(\mathrm{dd}, \mathrm{J}=6.4,2.6 \mathrm{~Hz}, 2 \mathrm{H}), 2.55(\mathrm{~s}, 1 \mathrm{H}), 2.09(\mathrm{t}, \mathrm{J}=2.6 \mathrm{~Hz}, 1 \mathrm{H}) .{ }^{13} \mathrm{C} \mathrm{NMR}\left(100 \mathrm{MHz}, \mathrm{CDCl}_{3}\right) \delta$ 151.914.7 (d, J = 244 Hz), $147.6(d, J=11 \mathrm{~Hz}), 138.8(\mathrm{~d}, \mathrm{~J}=3 \mathrm{~Hz}), 118.1$ (d, J = $7 \mathrm{~Hz}), 115.8$ (d, J = 19 $\mathrm{Hz}), 110.9$ (d, J = $2 \mathrm{~Hz}), 80.4,71.9,71.3,56.2,29.6$.

1-(4-fluoro-3-methylphenyl)but-3-yn-1-ol (3s) ${ }^{20} \mathrm{~d} 88 \%$ yield (78.4 mg), colorless oil. ${ }^{1} \mathrm{H}$ NMR (400 MHz, $\mathrm{CDCl}_{3}$ ) ठ 7.24-7.08 (m, 2H), $6.97(\mathrm{t}, \mathrm{J}=8.9 \mathrm{~Hz}, 1 \mathrm{H}), 4.81(\mathrm{t}, \mathrm{J}=6.3 \mathrm{~Hz}, 1 \mathrm{H}), 2.61(\mathrm{dd}, \mathrm{J}=6.3,2.4 \mathrm{~Hz}, 2 \mathrm{H})$, $2.45(\mathrm{~s}, 1 \mathrm{H}), 2.27(\mathrm{~s}, 3 \mathrm{H}), 2.08(\mathrm{~s}, 1 \mathrm{H}) .{ }^{13} \mathrm{C} \mathrm{NMR}\left(100 \mathrm{MHz}, \mathrm{CDCl}_{3}\right) \delta 160.9(\mathrm{~d}, \mathrm{~J}=243 \mathrm{~Hz}), 137.87,128.9$ $(\mathrm{d}, \mathrm{J}=2 \mathrm{~Hz}), 125.0,124.7(\mathrm{~d}, \mathrm{~J}=8 \mathrm{~Hz}), 114.9(\mathrm{~d}, \mathrm{~J}=22 \mathrm{~Hz}), 80.6,71.8,71.1,29.5,14.7(\mathrm{~d}, \mathrm{~J}=4 \mathrm{~Hz})$ 
2-(benzo[d][1,3]dioxol-4-yl)but-3-yn-1-ol (3t) ${ }^{20} \square$ 85\% yield (80.8 mg), colorless oil. ${ }^{1} \mathrm{H}$ NMR $(400 \mathrm{MHz}$, $\left.\mathrm{CDCl}_{3}\right)$ ઈ 6.95-6.89 (m, 1H), $6.84(\mathrm{t}, \mathrm{J}=7.8 \mathrm{~Hz}, 1 \mathrm{H}), 6.78(\mathrm{dd}, \mathrm{J}=7.6,1.0 \mathrm{~Hz}, 1 \mathrm{H}), 5.96(\mathrm{dd}, \mathrm{J}=9.2,1.1 \mathrm{~Hz}$, $2 \mathrm{H}), 4.98(\mathrm{dd}, \mathrm{J}=10.2,6.3 \mathrm{~Hz}, 1 \mathrm{H}), 2.84-2.58(\mathrm{~m}, 3 \mathrm{H}), 2.06(\mathrm{t}, \mathrm{J}=2.6 \mathrm{~Hz}, 1 \mathrm{H}) .{ }^{13} \mathrm{C} \mathrm{NMR}\left(100 \mathrm{MHz}, \mathrm{CDCl}_{3}\right)$ $\delta 147.4,144.1,124.1,121.8,119.3,108.2,101.0,80.5,70.9,68.3,27.6$.

1-(thiophen-2-yl)but-3-yn-1-ol (3u) ${ }^{20} \mathrm{\square} 88 \%$ yield (66.9 mg), colorless oil. ${ }^{1} \mathrm{H} \mathrm{NMR}\left(400 \mathrm{MHz}, \mathrm{CDCl}_{3}\right) \delta 7.32$ $7.22(\mathrm{~m}, 1 \mathrm{H}), 6.99$ (ddd, J = 11.1, 6.1, $2.5 \mathrm{~Hz}, 2 \mathrm{H}), 5.11(\mathrm{~d}, \mathrm{~J}=3.7 \mathrm{~Hz}, 1 \mathrm{H}), 2.79-2.68(\mathrm{~m}, 3 \mathrm{H}), 2.11$ (dd, J = 5.2, 2.6 Hz, 1H). ${ }^{13} \mathrm{C} \mathrm{NMR}\left(100 \mathrm{MHz}, \mathrm{CDCl}_{3}\right) \delta 146.2,126.7,125.0,124.2,80.1,71.5,68.5,29.5$.

1-(4-chloropyridin-2-yl)but-3-yn-1-ol (3v) ${ }^{20} \mathrm{d93} \%$ yield (84.1 mg), colorless oil. ${ }^{1} \mathrm{H}$ NMR (400 MHz, $\left.\mathrm{CDCl}_{3}\right) \delta 8.46(\mathrm{~d}, \mathrm{~J}=5.3 \mathrm{~Hz}, 1 \mathrm{H}), 7.49(\mathrm{~d}, \mathrm{~J}=1.7 \mathrm{~Hz}, 1 \mathrm{H}), 7.26(\mathrm{dd}, \mathrm{J}=5.4,2.0 \mathrm{~Hz}, 1 \mathrm{H}), 4.88(\mathrm{t}, \mathrm{J}=6.0 \mathrm{~Hz}$, $1 \mathrm{H}), 2.78-2.65(\mathrm{~m}, 2 \mathrm{H}), 2.06(\mathrm{t}, \mathrm{J}=2.6 \mathrm{~Hz}, 1 \mathrm{H}), 1.25(\mathrm{~s}, 1 \mathrm{H}) .{ }^{13} \mathrm{C} \mathrm{NMR}\left(100 \mathrm{MHz}, \mathrm{CDCl}_{3}\right) \delta 162.0,149.4$, $144.9,123.3,121.2,80.0,71.3,71.1,28.2$.

1-(pyridin-3-yl)but-3-yn-1-ol (3w) ${ }^{20} \mathrm{~d} 95 \%$ yield (69.9 mg), colorless oil. ${ }^{1} \mathrm{H}$ NMR $\left(400 \mathrm{MHz}, \mathrm{CDCl}_{3}\right) \delta 8.54$ (d, $\mathrm{J}=2.0 \mathrm{~Hz}, 1 \mathrm{H}), 8.47(\mathrm{dd}, \mathrm{J}=4.8,1.5 \mathrm{~Hz}, 1 \mathrm{H}), 7.79(\mathrm{dt}, \mathrm{J}=7.9,1.8 \mathrm{~Hz}, 1 \mathrm{H}), 7.33-7.26(\mathrm{~m}, 1 \mathrm{H}), 4.92(\mathrm{t}, \mathrm{J}=$ $6.4 \mathrm{~Hz}, 1 \mathrm{H}), 2.70-2.65(\mathrm{~m}, 2 \mathrm{H}), 2.08(\mathrm{t}, \mathrm{J}=2.6 \mathrm{~Hz}, 1 \mathrm{H}), 1.35-1.23(\mathrm{~m}, 1 \mathrm{H}) .{ }^{13} \mathrm{C} \mathrm{NMR}\left(100 \mathrm{MHz}, \mathrm{CDCl}_{3}\right) \delta$ $148.9,147.6,138.3,133.9,123.5,79.9,71.5,70.0,29.3$.

1-(quinolin-2-yl)but-3-yn-1-ol (3x) ${ }^{20}$ 口93\% yield (92.0 mg), colorless oil. ${ }^{1} \mathrm{H} \mathrm{NMR}\left(400 \mathrm{MHz}, \mathrm{CDCl}_{3}\right) \delta 8.19$ (d, $\mathrm{J}=8.5 \mathrm{~Hz}, 1 \mathrm{H}), 8.09(\mathrm{~d}, \mathrm{~J}=8.5 \mathrm{~Hz}, 1 \mathrm{H}), 7.85(\mathrm{~d}, \mathrm{~J}=8.1 \mathrm{~Hz}, 1 \mathrm{H}), 7.73(\mathrm{dd}, \mathrm{J}=8.4,1.4 \mathrm{~Hz}, 1 \mathrm{H}), 7.59-7.49(\mathrm{~m}$, $2 \mathrm{H}), 5.07(\mathrm{t}, \mathrm{J}=5.9 \mathrm{~Hz}, 1 \mathrm{H}), 2.80(\mathrm{ddd}, \mathrm{J}=5.9,2.5,1.7 \mathrm{~Hz}, 2 \mathrm{H}), 2.02(\mathrm{t}, \mathrm{J}=2.7 \mathrm{~Hz}, 1 \mathrm{H}), 1.25(\mathrm{~s}, 1 \mathrm{H}) .{ }^{13} \mathrm{C}$ $\operatorname{NMR}\left(100 \mathrm{MHz}, \mathrm{CDCl}_{3}\right) \delta 160.0,146.5,137.0,129.9,128.9,127.8,127.7,126.7,118.5,80.5,71.0,71.0$, 28.3.

1-(naphthalen-2-yl)but-3-yn-1-ol (3y) ${ }^{20} \square 94 \%$ yield (92.5 mg), colorless oil. ${ }^{1} \mathrm{H}$ NMR (400 MHz, $\left.\mathrm{CDCl}_{3}\right)$ ठ 7.87-7.70 (m, 4H), 7.56-7.36 (m, 3H), $4.98(\mathrm{t}, \mathrm{J}=6.3 \mathrm{~Hz}, 1 \mathrm{H}), 2.75(\mathrm{~s}, 1 \mathrm{H}), 2.69(\mathrm{dd}, \mathrm{J}=6.4,2.6 \mathrm{~Hz}$, 2H), $2.05(\mathrm{t}, \mathrm{J}=2.6 \mathrm{~Hz}, 1 \mathrm{H}) .{ }^{13} \mathrm{C} \mathrm{NMR}\left(100 \mathrm{MHz}, \mathrm{CDCl}_{3}\right) \delta 139.9,133.2,133.2,128.4,128.1,127.8,126.3$, $126.1,124.8,123.8,80.8,72.5,71.2,29.4$.

1-(naphthalen-1-yl)but-3-yn-1-ol (3z) ${ }^{20} 096 \%$ yield (94.1 mg), colorless oil. ${ }^{1} \mathrm{H}$ NMR (400 MHz, $\left.\mathrm{CDCl}_{3}\right) \delta 8.04(\mathrm{~d}, \mathrm{~J}=8.2 \mathrm{~Hz}, 1 \mathrm{H}), 7.89-7.83(\mathrm{~m}, 1 \mathrm{H}), 7.79(\mathrm{~d}, \mathrm{~J}=8.2 \mathrm{~Hz}, 1 \mathrm{H}), 7.69(\mathrm{~d}, \mathrm{~J}=7.2 \mathrm{~Hz}, 1 \mathrm{H}), 7.54-$ $7.44(\mathrm{~m}, 3 \mathrm{H}), 5.63(\mathrm{dd}, \mathrm{J}=8.2,4.2 \mathrm{~Hz}, 1 \mathrm{H}), 2.87$ (ddd, $\mathrm{J}=17.0,4.2,2.7 \mathrm{~Hz}, 1 \mathrm{H}), 2.73$ (ddd, J = 17.0, 8.2, 2.6 $\mathrm{Hz}, 2 \mathrm{H}), 2.12(\mathrm{t}, \mathrm{J}=2.6 \mathrm{~Hz}, 1 \mathrm{H}) .{ }^{13} \mathrm{C}$ NMR $\left(100 \mathrm{MHz}, \mathrm{CDCl}_{3}\right) \delta 137.8,133.8,130.2,129.1,128.5,126.3$, $125.7,125.4,123.0,122.8,81.0,71.3,69.3,28.7$.

1-(4-isopropylphenyl)-2-methyl-3ג5-buta-2,3-dien-1-ol (5a) ${ }^{20} 076 \%$ yield $(76.8 \mathrm{mg})$, colorless oil. ${ }^{1} \mathrm{H}$ NMR $\left(400 \mathrm{MHz}, \mathrm{CDCl}_{3}\right) \delta 7.30(\mathrm{~d}, \mathrm{~J}=8.0 \mathrm{~Hz}, 2 \mathrm{H}), 7.21(\mathrm{~d}, \mathrm{~J}=8.0 \mathrm{~Hz}, 2 \mathrm{H}), 5.07(\mathrm{~s}, 1 \mathrm{H}), 4.96-4.85(\mathrm{~m}, 2 \mathrm{H}), 2.90$ $(\mathrm{dt}, \mathrm{J}=13.8,6.9 \mathrm{~Hz}, 1 \mathrm{H}), 2.18(\mathrm{~s}, 1 \mathrm{H}), 1.58(\mathrm{t}, \mathrm{J}=3.0 \mathrm{~Hz}, 3 \mathrm{H}), 1.24(\mathrm{~d}, \mathrm{~J}=7.0 \mathrm{~Hz}, 6 \mathrm{H}) .{ }^{13} \mathrm{C} \mathrm{NMR}(100 \mathrm{MHz}$, $\left.\mathrm{CDCl}_{3}\right) \delta 204.6,148.6,139.2,126.6,126.5,102.7,77.9,74.5,33.9,24.0,14.7$. 


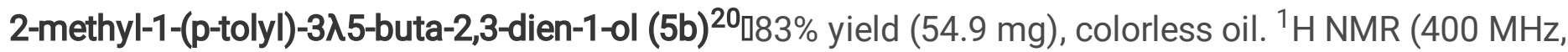
$\left.\mathrm{CDCl}_{3}\right) \delta 7.25(\mathrm{~d}, \mathrm{~J}=8.0 \mathrm{~Hz}, 2 \mathrm{H}), 7.15(\mathrm{~d}, \mathrm{~J}=7.9 \mathrm{~Hz}, 2 \mathrm{H}), 5.05(\mathrm{~s}, 1 \mathrm{H}), 4.96-4.82(\mathrm{~m}, 2 \mathrm{H}), 2.34(\mathrm{~s}, 3 \mathrm{H}), 2.30$ $(\mathrm{s}, 1 \mathrm{H}), 1.56(\mathrm{t}, \mathrm{J}=3.1 \mathrm{~Hz}, 3 \mathrm{H}) .{ }^{13} \mathrm{C}$ NMR $\left(100 \mathrm{MHz}, \mathrm{CDCl}_{3}\right) \delta 204.7,138.9,137.5,129.1,126.6,102.7$, $77.8,74.5,21.2,14.7$.

1-(4-fluorophenyl)-2-methyl-3ג5-buta-2,3-dien-1-ol (5c) ${ }^{20} \square 73 \%$ yield (73 mg), colorless oil. ${ }^{1} \mathrm{H}$ NMR (400 $\left.\mathrm{MHz}, \mathrm{CDCl}_{3}\right) \delta 7.34(\mathrm{dd}, \mathrm{J}=8.4,5.6 \mathrm{~Hz}, 2 \mathrm{H}), 7.03(\mathrm{t}, \mathrm{J}=8.7 \mathrm{~Hz}, 2 \mathrm{H}), 5.08(\mathrm{~s}, 1 \mathrm{H}), 4.93-4.86(\mathrm{~m}, 2 \mathrm{H}), 2.39(\mathrm{~s}$, $1 \mathrm{H}), 1.55(\mathrm{t}, \mathrm{J}=3.1 \mathrm{~Hz}, 3 \mathrm{H}) .{ }^{13} \mathrm{C} \mathrm{NMR}\left(100 \mathrm{MHz}, \mathrm{CDCl}_{3}\right) \delta 162.3(\mathrm{~d}, \mathrm{~J}=244 \mathrm{~Hz}), 137.5(\mathrm{~d}, \mathrm{~J}=3 \mathrm{~Hz}), 128.3$ $(\mathrm{d}, \mathrm{J}=8 \mathrm{~Hz}), 115.2(\mathrm{~d}, \mathrm{~J}=21 \mathrm{~Hz}), 102.6,78.1,77.4,74.0,14.5$.

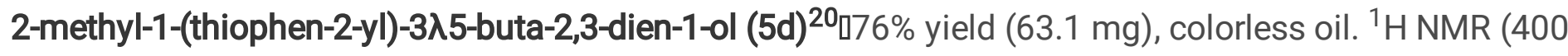

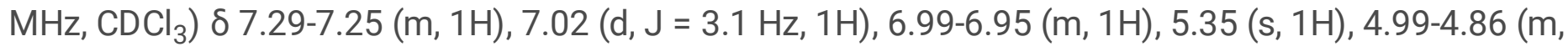
2H), $2.34(\mathrm{~d}, \mathrm{~J}=4.4 \mathrm{~Hz}, 1 \mathrm{H}), 1.69(\mathrm{t}, \mathrm{J}=3.0 \mathrm{~Hz}, 3 \mathrm{H}) .{ }^{13} \mathrm{C} \mathrm{NMR}\left(100 \mathrm{MHz}, \mathrm{CDCl}_{3}\right) \delta 204.4,146.1,126.6$, $125.2,125.0,102.6,78.6,70.7,14.7$.

\section{Conclusions}

In conclusion, we have established the first Cu-catalyzed, Mn-mediated propargylation and allenylation of aldehydes with propargyl bromides. The unique combination of the Cu catalyst and Mn powder present a novel and effective catalyst system in the preparation of homopropargylation alcohols and allenyl alcohols. The overall transformation is highly efficient with mild conditions, large substrate scope, and excellent chem-selectivity.

\section{Declarations}

Ethics approval and consent to participate

Not applicable.

Consent for publication

NA.

\section{Competing interests}

The authors declare no competing interests.

\section{Availability of data and materials}

All data generated or analyzed during this study are included in this published and its supplementary information files. 
The authors thank the National Natural Science Foundation of China (22161003) and the Project of Science and Technology of Xuzhou Government (No. KC16SG250)

Acknowledgement

NA

\section{Author details}

${ }^{1}$ Xuzhou Medical University, Tongshan Road 209, Xuzhou, 221004, China. ${ }^{2}$ School of Pharmaceutical Sciences, Gannan Medical University, Ganzhou 341000, China

Authors' contributions

ZRL contributed to the conception of the study. XY and OL performed the experiment. ZRL and YYC contributed to analysis and manuscript preparation. All authors read and approved the final manuscript.

\section{References}

1. (a) Alami, M.; Hamze, A.; Provot, O.; ACS Catal. 2019, 9, 3437. (b) Parker, K. D. J.; Fryzuk, M. D. Organometallics 2015, 34, 2037. (c) Corpas, J.; Mauleón, P.; Gómez Arrayá, M.; Carretero, J. C. ACS Catal. 2021, 11, 7513. (d) Gilmore, K.; Alabugin, I. V. Chem. Rev. 2011, 111, 6513. (e) Liu, L.; Ward, R. M.; Schomaker, J. M. Chem. Rev. 2019, 119, 12422. (f) Blieck, R.; Taillefer, M.; Monnier, F.; Chem. Rev. 2020, 120, 13545.

2. (a) Baran, P. S.; Shenvi, R. A. J. Am. Chem. Soc. 2006, 128, 14028. (b) Farahat, A. A.; Kumar, A.; Say, M.; Barghash, A. E. M.; Goda, F. E.; Eisa, H. M.; Wenzler, T.; Brun, R.; Liu, Y.; Mickelson, L.; Wilson, W. D.; Boykin, D. W. Bioorg. Med. Chem. 2010, 18, 557. (c) Grover, H. K.; Lebold, T. P.; Kerr, M. A. Org. Lett. 2011, 13, 220. (d) Zhang, W.; Ready, J. M. J. Am. Chem. Soc. 2016, 138, 10684. (e) Praveen, C.; Ananth, D. B. Bioorg. Med. Chem. Lett. 2016, 26, 2507. (f) Ma, S. Aldrichimica Acta 2007, 40, 91. (g) Brasholz, M.; Reissig, H.-U.; Zimmer, R. Acc. Chem. Res. 2009, 42, 45.

3. (a) Urgoita, G.; SanMartin, R.; Teresa Herrero, M.; Domínguez, E. ACS Catal. 2017, 7, 3050. (b) Chinchilla, R.; Nájera, C. Chem. Rev. 2014, 114, 1783. (c) Lin, W.; Ma, S. Org. Chem. Front. 2017, 4, 958. (d) Lu, T.; Lu, Z.; Ma, Z.-X.; Zhang, Y.; Hsung, R. P. Chem. Rev. 2013, 113, 4862. (e) Han, Y.; Ma, S. Org. Chem. Front. 2018, 5, 2680.

4. (a) Panek, J. S. Comprehensive Organic Synthesis, (Ed.), Pergamon Press, Oxford, 1991, 595. (b) Yamamoto, H. Comprehensive Organic Synthesis, (Ed.), Pergamon Press, Oxford, 1991, 81. (c) Yamaguchi, M. Main Group Metals in Organic Synthesis, (Eds.), Wiley-VCH, Weinheim, 2004, 307.

5. (a) Smith, M. B.; March, J. March's Advanced Organic Chemistry: Reactions, Mechanisms, and Structure, 6th (Ed), Jon Wiley and Sons, Inc.: New York, 2006; 752. (b) Yamamoto, H. In Comprehensive Organic Synthesis; (Ed.), Pergamon Press: New York, 1991; 81. 
6. (a) Araki, S.; Ito, H.; Butsugan, Y. J. Org. Chem. 1988, 53, 1833. (b) Isaac, M. B.; Chan, T.-H. J. Chem. Soc., Chem. Commun. 1995, 1003. (c) Miao, W.; Chung, L. W.; Wu, Y. D.; Chan, T. H. J. Am. Chem. Soc. 2004, 126, 13326. (d) T.-P. Loh, M.-J. Lin, K.-L. Tan, Tetrahedron Lett. 2003, 44, 507. (e) Haddad, T. D.; Hirayama, L. C.; Buckley, J. J.; Singaram, B. J. Org. Chem. 2012, 77, 889

7. Inoue, M.; Nakada, M. Org. Lett. 2004, 6, 2977.

8. Hojo, M.; Harada, H.; Ito, H.; Hosomi, A. Chem. Commun. 1997, 2077.

9. (a) Zha, Z. G.; Hui, A. L.; Zhou, Y. Q.; Miao, Q.; Wang, Z. Y.; Zhang, H. C. Org. Lett. 2005, 7, 1903. (b) Zha, Z. G.; Qiao, S.; Jiang, J. Y.; Wang, Y. S.; Miao, Q.; Wang, Z. Y. Tetrahedron 2005, 61, 2521.

10. (a) Hojo, M.; Sakuragi, R.; Okabe, S.; Hosomi, A. Chem. Commun. 2001, 357. (b) Lindstrom, U. M. Chem. Rev. 2002, 102, 2751.

11. (a) Kwon, J. S.; Pae, A. N.; Choi, K. I.; Koh, H. Y.; Kim, Y.; Cho, Y. S. Tetrahedron Lett. 2001, $42,1957$. (b) Han, Y.; Chi, Z.; Huang, Y.-Z. Synth. Commun. 1999, 29, 1287.

12. Bieber, L. W.; da Silva, M. F.; da Costa, R. C.; Silva, L. O. S. Tetrahedron Lett. 1998, 39, 3655.

13. (a) Tan, X. H.; Hou, Y. Q.; Liu, L.; Guo, Q. X. Chin. J. Chem. 2004, 22, 450. (b) Lee, A.S.-Y.; Chang, Y.-T.; Wang, S.-H.; Chu, S.-F. Tetrahedron Lett. 2002, 43, 8489. (c) Fandrick, D. R.; Reeves, J. T.; Bakonyi, J.; Nyalapatla, P. R. J. Org. Chem. 2013, 78, 3592.

14. (a) Iseki, K.; Kuroki, Y.; Kobayashi, Y. Tetrahedron: Asymmetry 1998, 9, 2889. (b) Nüchter, M.; Ondruschka, B.; Bonrath, W.; Gum, A. Green Chem. 2004, 6, 128.

15. Evans, D. A.; Sweeney, Z. K.; Rovis, T.; Tedrow, J. S.; J. Am. Chem. Soc. 2001, 123, 12095.

16. Marshall, J. A.; Gung, B. W.; Grachan, M. L. Synthesis and Reactions of Allenylmetal Compounds. In Modern Allene Chemistry, (Ed.) Wiley-VCH: Weinheim, 2004; 493.

17. (a) Wendlandt, A. E.; Suess, A. M.; Stahl, S. S. Angew. Chem. Int. Ed. 2011, 50, 11062. (b) McCann, S. D.; Stahl, S. S. Acc. Chem. Res. 2015, 48, 1756.

18. (a) Allen, S. C.; Walvoord, R. R.; Padilla-Salinas, R.; Kozlowski, M. C. Chem. Rev. 2013, 113, 6234. (b) Guo, X.-X.; Gu, D.-W.; Wu, Z.; Zhang, W. Chem. Rev. 2015, 115, 1622.

19. (a) Crossley, S. W. M.; Obradors, C.; Martinez, R. M.; Shenvi, R. A. Chem. Rev. 2016, 116, 8912. (b) Irrgang, T.; Kempe, R. Chem. Rev. 2019, 119, 2524.

20. Mori-Quiroz, L. M., Maloba, E. W., \& Maleczka, R. E. Org. Lett. 2021, 23, 5724-5728.

\section{Tables}

Tables 1-3 are available in the Supplementary Files section.

\section{Figures}


(a) Synthesis of homopropargyl or allenyl alcohol via metallotropic rearrangement:

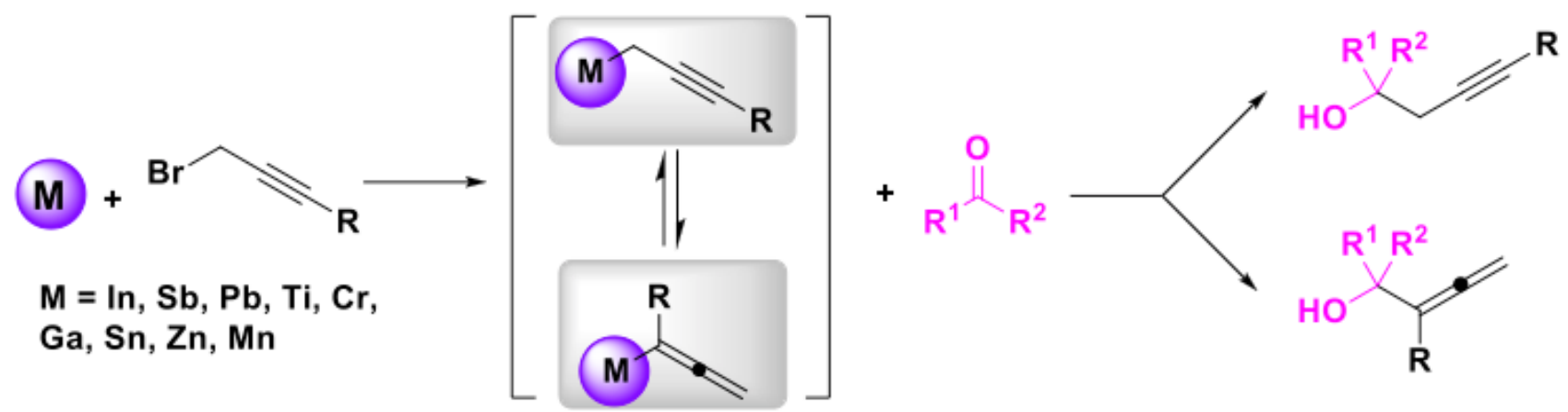

(b) Cu-catalyzed and Mn-mediated propargylation and allenylation (this work):

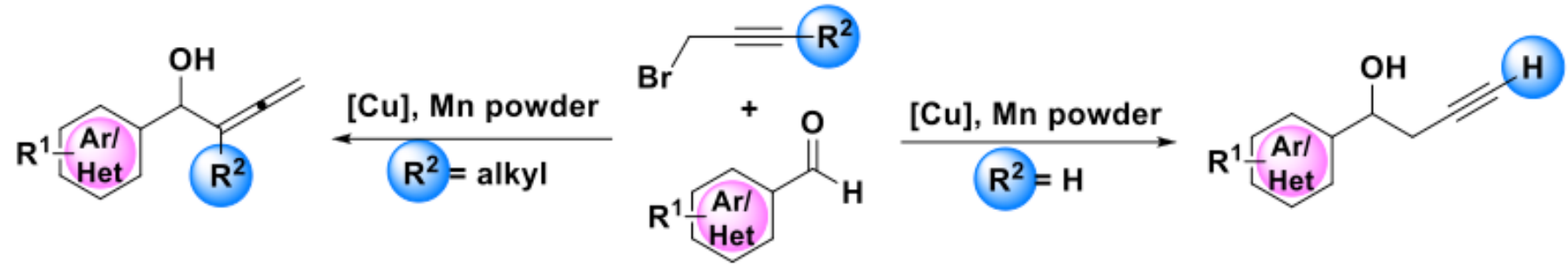

High yield Mild reaction condition Good chemoselectivity Wide substrates scope

Figure 1

Previous studies and our concept.

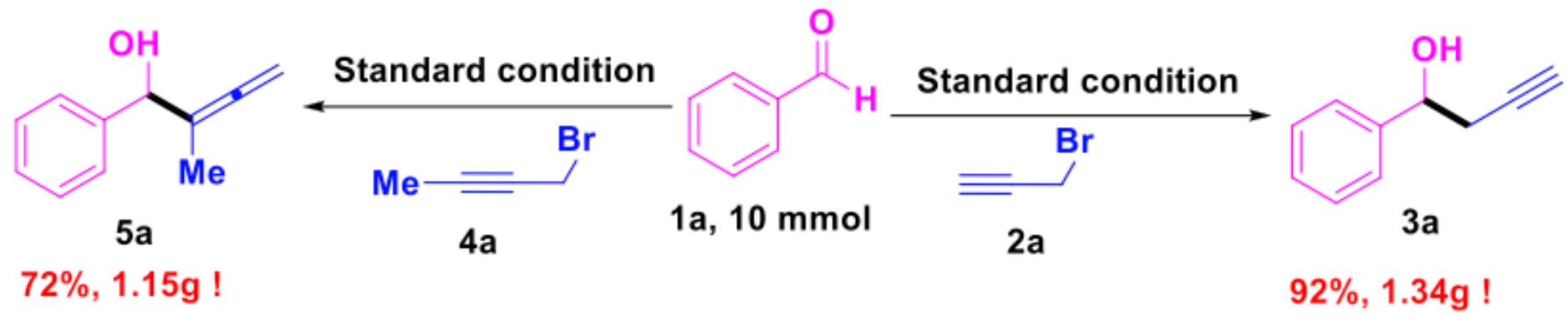

Figure 2

Gram-scale synthesis of $3 a$ and $5 a$

\section{Supplementary Files}

This is a list of supplementary files associated with this preprint. Click to download.

- BMC20211015SI.doc 
- Table1.docx

- Table2.docx

- Table3.docx 